\title{
Dose-response relationships between sedentary behaviour and the metabolic syndrome and its components
}

\author{
Keith P. Gennuso • Ronald E. Gangnon • Keith M. Thraen-Borowski • \\ Lisa H. Colbert
}

Received: 9 October 2014 / Accepted: 29 October 2014 / Published online: 5 December 2014

(C) Springer-Verlag Berlin Heidelberg 2014

\begin{abstract}
Aims/hypothesis The aim of this study was to examine the relationship among sedentary behaviour (SB) and the metabolic syndrome and its components by age, moderate-tovigorous physical activity (MVPA) and sex.

Methods A cross-sectional analysis was performed on 20032006 National Health and Nutrition Examination Survey data from 5,076 adults aged $\geq 18$ years (mean $\pm \mathrm{SD}=43.8 \pm 19.5$ ). SB was measured using ActiGraph accelerometers worn for 1 week and defined as $<100$ counts $/ \mathrm{min}$. Metabolic syndrome was defined using the Adult Treatment Panel III criteria. Natural cubic spline logistic regression models were used to estimate the odds of meeting criteria for the metabolic syndrome and its components by total daily SB time and breaks in SB. Statistical interactions between SB and age, sex and MVPA were explored.

Results The prevalence of the metabolic syndrome was $19 \%$ and the average daily SB time was $8.1 \pm 2.8 \mathrm{~h}$, with $90 \pm 25$ breaks/day. The relationship between daily SB time and the metabolic syndrome was linear and characterised by an OR of 1.09 (95\% CI 1.01, 1.18) for each hour of SB. Total SB was associated with the following components: high triacylglycerol,
\end{abstract}

K. P. Gennuso $(\bowtie)$

University of Wisconsin Population Health Institute, 575C Warf

Office Building, 610 Walnut St., Madison, WI 53726, USA

e-mail: gennuso@wisc.edu

R. E. Gangnon

Department of Population Health Sciences, University of

Wisconsin-Madison, Madison, WI, USA

R. E. Gangnon

Department of Biostatistics and Medical Informatics, University of

Wisconsin-Madison, Madison, WI, USA

K. M. Thraen-Borowski $\cdot$ L. H. Colbert

Department of Kinesiology, University of Wisconsin-Madison, Madison, WI, USA low HDL-cholesterol and high fasting glucose. All three associations were modified by MVPA level. No relationship between breaks in SB and the metabolic syndrome was found. Conclusions/interpretation There appears to be no SB threshold at which the risk of the metabolic syndrome is elevated. Therefore, an effort should be made to maintain low levels of total time spent in SB and so lessen the risk of the metabolic syndrome.

Keywords Epidemiology $\cdot$ Metabolic syndrome X $\cdot$ Physical activity $\cdot$ Sedentary lifestyle

\begin{tabular}{ll}
\multicolumn{2}{l}{ Abbreviations } \\
MVPA & Moderate-to-vigorous physical activity \\
NHANES & National Health and Nutrition Examination \\
& Survey \\
SB & Sedentary behaviour
\end{tabular}

\section{Introduction}

Adults in the USA spend most of their day being sedentary. According to accelerometry data for a nationally representative sample from the 2003-2004 measurement cycle of the National Health and Nutrition Examination Survey (NHANES) [1], approximately $8 \mathrm{~h} /$ day are spent in sedentary behaviour (SB), that is, activities such as sitting and reclining that do not increase energy expenditure substantially [2]. Over the last 15 years, numerous studies have examined the health consequences of spending too much time in SB. A number of longitudinal studies have provided evidence for an association between SB (or proxy measures such as television viewing) and various health outcomes in adults, including increased risk of all-cause and cardiovascular disease related mortality, several site-specific cancers and type 2 diabetes [3]. 
Importantly, these relationships remain after controlling for and investigating effect modification by participation in moderate-to-vigorous physical activity (MVPA).

Recently, there has been increased interest in the association between SB and the metabolic syndrome, which comprises a cluster of factors, including reduced HDL-cholesterol and elevated waist circumference, triacylglycerol, BP and fasting glucose, that are known to promote or increase the risk of developing cardiovascular disease and type 2 diabetes [4]. A number of recent studies have explored this association, including one meta-analytical review [5]. The review examined ten cross-sectional studies with an overall sample size of 21,393 and a $26.1 \%$ prevalence of the metabolic syndrome. Those in the highest quantiles of SB had a $73 \%$ increased odds (OR 1.73; 95\% CI 1.55, 1.94) of the metabolic syndrome compared with those in the lowest quantiles. This relationship persisted following a sensitivity analysis including only studies that controlled for participation in physical activity. However, one area lacking in this field of inquiry is a dedicated examination of the dose-response relationship between SB and the metabolic syndrome.

Most dose-response studies have used study-specific quantiles of SB, often assessed using self-reported sitting time or television viewing as their SB measure. Studies with more accurate SB measures, such as accelerometry [6,7], or more informative methods to address the dose-response, such as spline regression analysis [8], may be better suited to addressing this issue. However, as far as we are aware, no studies have combined both objective activity measurement and spline regression analysis to explore the association between $\mathrm{SB}$ and the metabolic syndrome. Therefore, we examined the dose-response relationship between SB, the metabolic syndrome and its components using a combination of accelerometry data from a representative sample of US adults and spline regression analysis to account for and more accurately describe the potential for non-linearity. We hypothesised that there would be a certain amount of SB that, once accumulated, would be related to an increased odds of the metabolic syndrome and its components, and that this amount would vary by sex, age and amount of MVPA.

\section{Methods}

Study population This study was conducted using data from NHANES, a continual US population-based survey conducted by the National Center for Health Statistics that consists of an in-home interview and a comprehensive medical examination. Each year, NHANES recruits roughly 5,000 noninstitutionalised civilians using a multistage probability design with sampling domains defined by race, sex, age and income [9]. The current study's sample was drawn from the 8,363 adults aged 18 years or older who participated in an objective assessment of ambulatory activity substudy during the 2003 2004 and 2005-2006 measurement cycles. The study was approved by the National Center for Health Statistics Research Ethics Review Board and documented consent was obtained from all participants.

$S B$ and physical activity assessment Participants of the ambulatory activity substudy wore an ActiGraph AM-7164 accelerometer (ActiGraph, Fort Walton Beach, FL, USA) for 1 week on an elastic belt over the right hip during all waking hours. Data in the form of activity counts per $1 \mathrm{~min}$ epoch were downloaded from the monitors upon receipt by mail. An explanation of the methods used for estimating wear time and time spent in SB and MVPA from the downloaded data has been described [10]. Briefly, monitor wear time was determined by subtracting non-wear time (i.e. periods of at least 60 consecutive minutes of no activity with an allowance for 2 consecutive minutes of observations between 1 and 100 counts) from the total daily observation time. Activity of between 1 and 100 counts/min was considered SB, while activity of at least 760 counts/min [11] with an allowance for interruptions of 1 or 2 min below threshold was considered MVPA. A valid day consisted of at least $600 \mathrm{~min}(10 \mathrm{~h})$ of wear without excessive counts ( $>20,000$ counts). Laboratorybased studies typically require 4 or more days of valid data for inclusion in their study sample. While this would have been preferable, the logistical challenges of using accelerometry in large-scale population-based surveys make this level of compliance difficult to obtain; therefore, we required at least 1 valid day to be included in the current analysis, a method consistent with the original examinations of NHANES data for MVPA [12] and SB time [1], so as to retain as many participants as possible and avoid selection bias.

Outcome and covariate assessment The primary outcome of interest in this study was the metabolic syndrome. We defined the metabolic syndrome according to the Third Report of the Expert Panel on Detection, Evaluation, and Treatment of High Blood Cholesterol in Adults (Adult Treatment Panel III) [4]. According to these guidelines, the metabolic syndrome is the presence of three or more of the following: (1) waist circumference $\geq 102 \mathrm{~cm}$ for men and $\geq 88 \mathrm{~cm}$ for women; (2) serum triacylglycerol level of $\geq 1.69 \mathrm{mmol} / \mathrm{l}$ or drug treatment for elevated triacylglycerol; (3) HDL-cholesterol level of $<1.04 \mathrm{mmol} / \mathrm{l}$ for men and $<1.29 \mathrm{mmol} / 1$ for women or drug treatment for reduced HDL; (4) fasting glucose level $\geq 5.55 \mathrm{mmol} / 1$ or use of glucose-lowering medications (insulin or oral agents); or (5) systolic $\mathrm{BP} \geq 130 \mathrm{mmHg}$ and/or diastolic $\mathrm{BP} \geq 85 \mathrm{mmHg}$ or the use of antihypertensive medications. Waist circumference was measured twice using a steel tape at the iliac crest to the nearest $0.1 \mathrm{~cm}$. Up to four BP measurements were obtained, with all but the first being averaged for the final value [13]. Twelve-hour fasting blood samples were 
drawn from a subsample of 3,850 individuals to assess HDL-cholesterol, triacylglycerol, and plasma glucose according to standard procedures (see [14] for details of the laboratory methods). Glucose-lowering and antihypertensive medication use was assessed by questionnaire. Information regarding drug treatment for elevated triacylglycerol and reduced HDL were unavailable. Similar to the methods proposed by Bankoski et al, [7] participants who had at least three positive metabolic syndrome criteria were considered to have the metabolic syndrome, people who had at least three negative metabolic syndrome criteria were considered not to have the metabolic syndrome and the rest were considered to be of unknown status. Using these criteria, we were able to confidently classify the metabolic syndrome status of 5,864 individuals. The remaining 2,499 individuals with unknown metabolic syndrome status were removed from the final sample. Finally, a further 788 were excluded for missing covariates to give a final analytical sample of 5,076.

Statistical analysis All analyses were performed using SAS version 9.3 (SAS Institute, Cary, NC, USA). To account for the complex, multistage probability sampling design, PROC SURVEY procedures were used with weights provided by the NHANES. To address the primary aim, the relationship of time spent in SB and breaks in SB with the metabolic syndrome and each of its components, we initially used natural cubic spline logistic regression models. Four knots were chosen at the minimum, maximum, and 25th and 75 th percentiles of time spent in SB and breaks in SB; the significance of non-linearity was examined by a likelihood ratio test to comparing a model with the linear term only and a model with both linear and the cubic spline terms. Where there was evidence for a linear relationship, logistic regression models were used in place of the spline models. Covariates for all regression analyses included age, sex, race/ethnicity, education, marital status, family income and current smoking status. Average weekly MVPA, total SB time and accelerometer wear time were also controlled for where appropriate and were derived from accelerometry data, as previously described.

To address the secondary hypotheses, the analyses were then stratified by age, sex and physical activity status, and tests for significant statistical interactions were performed using cross-product terms in the regression models. Age and time spent in MVPA were divided into the following respective categories: $18-39,40-59$ and $\geq 60$ years; and $<75$, 75-149, 150-299 and $\geq 300 \mathrm{~min} /$ week. There was evidence for a statistically significant interaction between SB and MVPA for several of the metabolic syndrome components; therefore, separate analyses were performed by MVPA level. Where the interaction was not statistically significant, estimates for combined MVPA groups are presented.

\section{Results}

Participant summary Full information about participant characteristics can be found in Table 1. Overall, the entire study sample had an average age of $43.8 \pm 19.5($ mean \pm SD) years, had a BMI of $27.9 \pm 6.3 \mathrm{~kg} / \mathrm{m}^{2}$ and was $54 \%$ female. The sample was also most likely to be non-Hispanic white (52\%) and married (64\%). Those with more than $8 \mathrm{~h}$ of total daily SB appeared to be older and more highly educated, but similar in other demographic variables to those with less than $8 \mathrm{~h} /$ day. The prevalence of the metabolic syndrome in the whole sample was $19 \%$ (Table 2). The median number of valid days that participants wore the activity monitors was 6 (4-7; interquartile range). The average daily SB time was $8.2 \pm 2.3 \mathrm{~h}$, with $90 \pm 19$ breaks/day and $179 \pm 183 \mathrm{~min} /$ week MVPA. Those with more than $8 \mathrm{~h} /$ day SB appeared to have a higher

Table 1 Participant characteristics by daily SB time

\begin{tabular}{llll}
\hline Participant characteristics & SB $<8 \mathrm{~h}$ & $\mathrm{SB} \geq 8 \mathrm{~h}$ & Total \\
\hline$n$ & 2,496 & 2,580 & 5,076 \\
Female & $1,432(57)$ & $1,325(51)$ & $2,757(54)$ \\
Age (years) & & & \\
$\quad$ 18-39 & $1,238(50)$ & $829(32)$ & $2,067(41)$ \\
$\quad$ 40-59 & $805(32)$ & $732(28)$ & $1,537(30)$ \\
$\quad$ 60+ & $453(18)$ & $1,019(39)$ & $1,472(29)$ \\
BMI & & & \\
$\quad$ Underweight & $39(2)$ & $65(3)$ & $104(2)$ \\
$\quad$ Normal & $812(33)$ & $777(30)$ & $1,589(31)$ \\
$\quad$ Overweight & $838(34)$ & $873(34)$ & $1,711(34)$ \\
$\quad$ Obese & $807(32)$ & $865(34)$ & $1,672(33)$ \\
Income & & & \\
$\quad<$ 20k & $720(29)$ & $685(27)$ & $1,405(28)$ \\
$\quad$ \$20k-\$44k & $829(33)$ & $760(29)$ & $1,589(31)$ \\
$\quad$ \$45k-\$75k & $496(20)$ & $532(21)$ & $1,028(20)$ \\
$\quad$ > $75 \mathrm{k}$ & $451(18)$ & $603(23)$ & $1,054(21)$ \\
Education & & & \\
$\quad<$ High school & $785(31)$ & $543(21)$ & $1,328(26)$ \\
$\quad$ High school & $652(26)$ & $556(22)$ & $1,208(24)$ \\
$\quad>$ High school & $1,059(42)$ & $1,481(57)$ & $2,540(50)$ \\
Marital status & & & \\
$\quad$ Married & $1,667(67)$ & $1,585(61)$ & $3,252(64)$ \\
$\quad$ Single & & & \\
$\quad$ Divorced & $537(22)$ & $675(26)$ & $1,212(24)$ \\
Race/ethnicity & $292(12)$ & $320(12)$ & $612(12)$ \\
$\quad$ Non-Hispanic white & $1,164(47)$ & $1,469(57)$ & $2,633(52)$ \\
$\quad$ Non-Hispanic black & $465(19)$ & $538(21)$ & $1,003(20)$ \\
$\quad$ Mexican American & $686(27)$ & $389(15)$ & $1,075(21)$ \\
$\quad$ Other & & & $365(7)$ \\
\hline
\end{tabular}

Values presented as $n(\%)$

a The marital status category of 'single' includes widows 
Table 2 The metabolic syndrome, its components, SB and MVPA by daily SB time

\begin{tabular}{|c|c|c|c|}
\hline Variable & $<8 \mathrm{~h}$ & $\geq 8 \mathrm{~h}$ & Total \\
\hline$n$ & 2,496 & 2,580 & 5,076 \\
\hline Metabolic syndrome & 349 (14) & $640(25)$ & 989 (19) \\
\hline Large waist circumference ${ }^{a}$ & $343(14)$ & $575(23)$ & $918(18)$ \\
\hline High triacylglycerol $^{\mathrm{b}}$ & $529(34)$ & $630(36)$ & $1,159(35)$ \\
\hline Low $\mathrm{HDL}^{\mathrm{c}}$ & $204(8)$ & $310(12)$ & $514(10)$ \\
\hline High glucose ${ }^{d}$ & $634(40)$ & $913(49)$ & $1,547(45)$ \\
\hline High $\mathrm{BP}^{\mathrm{e}}$ & $610(25)$ & $1,050(41)$ & $1,660(33)$ \\
\hline Total daily SB time (h/day) & $6.6(5.6-7.3)$ & $9.4(8.7-10.6)$ & $8.0(6.7-9.5)$ \\
\hline Breaks (number/day) & $89(78-100)$ & $91(77-104)$ & $90(77-102)$ \\
\hline MVPA (min/week) & $154(70-286)$ & $82(29-181)$ & $116(46-230)$ \\
\hline
\end{tabular}

Values for categorical and continuous variables are $n(\%)$ and median (25-75\%), respectively

${ }^{\text {a }}$ Frequency missing $=57 ;<8 \mathrm{~h} 22, \geq 8 \mathrm{~h} 35$

${ }^{\mathrm{b}}$ Frequency missing $=1,761 ;<8$ h $940, \geq 8$ h 821

${ }^{\mathrm{c}}$ Frequency missing $=13 ;<8 \mathrm{~h} 6, \geq 8 \mathrm{~h} 7$

${ }^{\mathrm{d}}$ Frequency missing $=1,615 ;<8 \mathrm{~h} 891, \geq 8 \mathrm{~h} 724$

${ }^{\mathrm{e}}$ Frequency missing $=78 ;<8$ h $34, \geq 8$ h 44

prevalence of the metabolic syndrome, a large waist circumference, high blood glucose, high BP and less weekly MVPA.

Linearity Our primary hypothesis was that there would be a certain amount of SB (total time and breaks) that, once accumulated, would be related to an increased odds of meeting the criteria for the metabolic syndrome and its components. This hypothesis had two premises: (1) that SB is significantly related to the metabolic syndrome and its components; and (2) that the curves that describe these relationships are non-linear. Regarding the first premise, we found a statistically significant relationship between total time spent in SB (h/day) and the metabolic syndrome and several of its components. The results of the logistic regression models describing these relationships will be presented in the following section. However, we did not find an association between breaks in SB and any of the dependent variables (data not shown). Regarding the second premise, we did not find any evidence for non-linear relationships for total SB time and breaks with the metabolic syndrome and its components. To illustrate this point, we plotted both the linear and natural cubic spline curves describing the relationship between the amount of SB time and OR for the metabolic syndrome (Fig. 1). The $p$ value for the linear term describing this relationship was 0.04 and for the non-linear term was 0.50 .

Total time spent in $S B$ As previously stated, the total time spent in SB was significantly related to the metabolic syndrome (Table 3). We found an OR of 1.09 (95\% CI 1.01, 1.18) for a $1 \mathrm{~h}$ increase in daily SB time. However, SB time was not associated with a large waist circumference (OR 1.02; 95\% CI $0.93,1.12$ ). To address our secondary hypothesis, we examined whether associations of SB with the metabolic syndrome and its components were modified by sex, age and MVPA. We did not find evidence of effect modification by these factors for the metabolic syndrome, as depicted in Fig. 2. Nor did we find effect modification by age or sex for any of the components; however, the associations between SB time and several components of the metabolic syndrome appeared to be modified by MVPA level (Table 3). Statistically significant interactions were found between SB and MVPA for triacylglycerol $(p=0.04)$, HDL-cholesterol $(p=0.01)$, fasting blood glucose $(p<0.001)$ and $\mathrm{BP}(p=0.01)$. The ORs associated with a $1 \mathrm{~h}$ increase in SB time for having high triacylglycerol, low HDLcholesterol and high fasting blood glucose were only statistically significant for the highest two quartiles of MVPA. That is, there was a $13 \%$ (OR $1.13 ; 95 \%$ CI $1.03,1.23)$ and $21 \%$ (OR 1.21; 95\% CI 1.08, 1.35) increased odds of having high

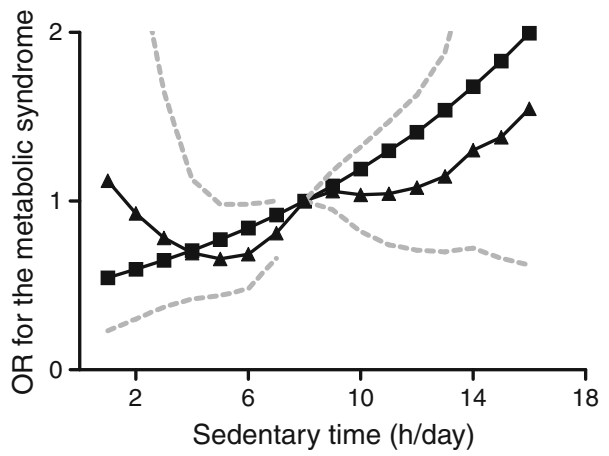

Fig. 1 Associations between daily sedentary time and ORs for the metabolic syndrome using linear (squares) and natural cubic spline (triangles) logistic regression models. Dotted lines indicate the $95 \%$ CI for the natural cubic spline model. $p$ values for the linear and cubic terms were 0.04 and 0.50 , respectively. Both models were adjusted for age, sex, education, family income, race/ethnicity, marital status, current smoking status, monitor wear time and MVPA 
Table 3 OR associated with a $1 \mathrm{~h} /$ day increase in SB time by MVPA level and in combined sample regardless of MVPA

\begin{tabular}{lllllll}
\hline & $<75 \mathrm{~min} /$ week & $75-149 \mathrm{~min} /$ week & $150-299 \mathrm{~min} /$ week & $\geq 300 \mathrm{~min} /$ week & $P_{\text {interaction }}{ }^{\mathrm{a}}$ & Combined $^{\mathrm{b}}$ \\
\hline Metabolic syndrome & $1.07(0.98,1.17)$ & $1.02(0.86,1.21)$ & $1.14(1.02,1.28)$ & $1.15(1.01,1.33)$ & 0.29 & $1.09(1.01,1.18)$ \\
High waist circumference & $0.95(0.85,1.07)$ & $1.01(0.86,1.19)$ & $1.07(0.95,1.20)$ & $1.03(0.91,1.16)$ & 0.48 & $1.02(0.93,1.12)$ \\
High triacylglycerol & $1.05(0.98,1.13)$ & $1.08(0.95,1.22)$ & $1.13(1.03,1.23)$ & $1.21(1.08,1.35)$ & 0.04 & \\
Low HDL-cholesterol & $0.93(0.84,1.03)$ & $1.02(0.84,1.23)$ & $1.19(1.05,1.34)$ & $1.23(1.05,1.44)$ & 0.01 \\
High fasting glucose & $1.04(0.95,1.14)$ & $0.90(0.82,0.99)$ & $1.06(0.97,1.17)$ & $1.13(1.01,1.27)$ & $<0.001$ \\
High BP & $1.03(0.93,1.14)$ & $0.85(0.74,0.97)$ & $1.04(0.91,1.20)$ & $0.86(0.76,0.98)$ & 0.01 \\
\hline
\end{tabular}

Data are presented as OR $(95 \% \mathrm{CI})$

${ }^{\text {a }}$ Adjusted for age, sex, education, family income, race/ethnicity, marital status, current smoking status and monitor wear time

${ }^{\mathrm{b}}$ Also adjusted for MVPA

triacylglycerol, and a 19\% (OR 1.19; 95\% CI 1.05, 1.34) and $23 \%$ (OR 1.23; 95\% CI 1.05, 1.44) increased odds of having low HDL-cholesterol with each hour of SB time for those who accumulated 150-300 and $\geq 300 \mathrm{~min} /$ week of MVPA, respectively. Similarly, we found an increased odds of $13 \%$ (OR 1.13; $95 \%$ CI 1.01, 1.26) for having high fasting blood glucose with each hour of SB time for those who accumulated $\geq 300 \mathrm{~min} /$ week of MVPA. The pattern describing the relationship between SB time and the odds of having high BP by MVPA level is less consistent. There was a counterintuitive reduction in odds found for the second (OR 0.84; 95\% CI 0.73, 0.96) and fourth (OR 0.87; 95\% CI 0.77, 0.98) quartiles of MVPA.

\section{Discussion}

We examined the dose-response relationship between SB and the metabolic syndrome and its components in a large nationally representative sample of US adults with objectively measured activity data. Our main finding was that total daily time spent in SB was associated with a linear increase in the odds of having the metabolic syndrome, without influence from any tested effect modifiers. This contradicted our hypothesis that there would be a certain threshold of SB necessary to increase the odds of the metabolic syndrome, which would vary by age, sex and amount of MVPA. We also found the total daily amount of SB to be linearly related to increased odds of meeting the criteria for the high triacylglycerol, low HDL-cholesterol and high fasting blood glucose components of the metabolic syndrome. Furthermore, this relationship seemed to be modified by weekly participation in MVPA. Conversely, we did not find evidence for a relationship between breaks in SB and increased odds of meeting the criteria for the metabolic syndrome or any of its components.

We are unaware of other studies involving similar analyses (i.e. which treated both the metabolic syndrome and its components as dichotomous variables), thus making direct comparisons difficult. However, parallels can be drawn with studies that have treated the components as continuous variables. Regarding breaks in SB, we are aware of one study that consistently found significant linear relationships between SB breaks and measures of insulin resistance and lipid variables [15]; however, our study corroborates the findings of most other studies on this subject [16-19], which have not found similar relationships. In the previously mentioned studies, one component of the metabolic syndrome consistently found to be related to SB breaks is waist circumference. We could not confirm a similar statistically significant relationship in the current study. We did, however, find a similar trend, characterised by a $6 \%$ reduction in the odds of meeting the criteria for high waist circumference with every 10 breaks, which almost reached significance (OR 0.94; $95 \%$ CI $0.85,1.04)$.

Findings from the current study extend the body of literature regarding total time spent in SB and markers of cardiometabolic health. Our evidence for a $9 \%$ increase in the odds of having the metabolic syndrome with each additional hour of daily SB is consistent with the findings of a previous metaanalytical review on the subject [5], although with a lesser magnitude. This is to be expected, though, because we examined SB as a continuous variable while the review reported differences between the highest and lowest quantiles of SB. When our data was analysed in a similar fashion for comparative purposes, we found a $58 \%$ (OR $1.58 ; 95 \%$ CI 1.01, 2.48) increased odds of the metabolic syndrome in the highest quartile of SB $(>9.49 \mathrm{~h})$ compared with the lowest $(<6.70 \mathrm{~h})$. Our findings regarding the components of the metabolic syndrome in relation to total SB time are also consistent with published reports. Previous studies have demonstrated significant associations between daily SB and triacylglycerol [6, 16, 17], HDL-cholesterol [16, 18] and fasting plasma glucose [17].

The relationships between total SB time and the components of high triacylglycerol, low HDL-cholesterol and high fasting glucose were modified by participation in MVPA. Interestingly, significantly increased odds of meeting the criteria for these components were found only in those in the 

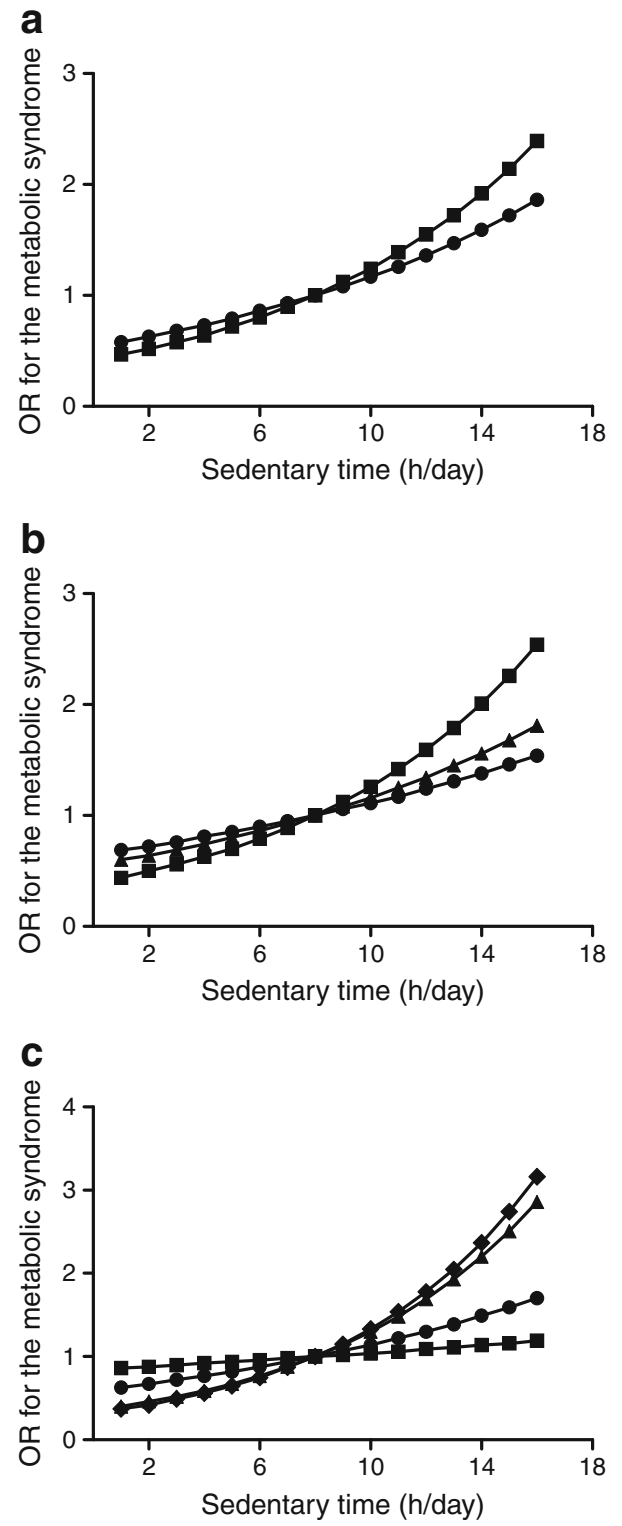

Fig. 2 Associations between daily sedentary time and ORs for the metabolic syndrome by (a) sex, (b) age and (c) MVPA. (a) Circles, men; squares, women; $p$ value for the interaction between sex and $\mathrm{SB}=0.53$. (b) Circles, 18-39 years; squares, 40-59 years; triangles, $\geq 60$ years; $p$ value for the interaction between age and $\mathrm{SB}=0.46$. (c) Circles, $<75 \mathrm{~min} /$ week; squares, 75-149 $\mathrm{min} /$ week; triangles, $150-299 \mathrm{~min} /$ week; diamonds, $\geq 300 \mathrm{~min} / \mathrm{week} ; p$ value for the interaction between MVPA and $\mathrm{SB}=0.28$

highest quartiles of MVPA. It is established that regular participation in activity of at least moderate intensity reduces the risk of the metabolic syndrome [20]. Evidence from crosssectional studies suggests that $120-180 \mathrm{~min} /$ week of MVPA is the minimum amount associated with lower rates of the metabolic syndrome [21-23]. Extending these findings to the current study might help explain why only in those with 150 or more min/week of MVPA was there an association with SB. It is conceivable that the lack of adequate MVPA in the lower two quartiles outweighed the effect of varying amounts of SB on the odds of meeting criteria for those components. Meanwhile, in the upper quartiles, attainment of adequate MVPA provided an opportunity for the effect of SB. We also found an effect modification by MVPA on the relationship between total SB and high BP; however, the lack of a linear trend makes us suspect that the association is spurious.

The novelty of SB research means that there is a lack of studies describing the effect of prolonged free-living SB on human physiology. Therefore, any insight into potential mechanisms behind the association between SB and the metabolic syndrome must be gleaned from animal studies or from human studies using more extreme models of SB. For instance, lipoprotein lipase, an enzyme essential for lipoprotein-derived fatty acid uptake [24] and whose dysfunction is associated with dyslipidaemia [25] and hypertension [26], is reduced in both concentration and activity by hindlimb unloading in rats [27]. In addition, the effect of prolonged SB has been demonstrated on muscle GLUT proteins, which are integral for insulin- and exercise-stimulated uptake of plasma glucose. A study of spinal cord injury by Megeney et al found reductions in GLUT content and glucose uptake within 3 days of muscle denervation [28]. Our finding of effect modification by MVPA on the relationship between SB and the components of triacylglycerol, HDL-cholesterol and fasting glucose implies that SB and MVPA may exert their effects through similar pathways for some biomarkers but different pathways for others. This notion is supported in a review of several studies by Hamilton et al [29], who demonstrated that the expression of some genes important for preventing diabetes and other metabolic risks is resistant to being restored after $12 \mathrm{~h}$ of inactivity, while the expression of others is not. In particular, a study examining the activity of lipid phosphate phosphatase-1 (LPP1) suggested that gene expression may be suppressed in response to chronic sitting despite participation in $1 \mathrm{~h}$ /day of vigorous exercise [30].

This study adds to the body of literature concerning the dose-response relationship between SB and the metabolic syndrome. A major strength of this study is the use of accelerometry data from a large, nationally representative sample of US adults. This enabled the concurrent measurement of SB and MVPA without relying on participant recall. Another strength was our consideration of the interaction between SB and MVPA. Most studies treat MVPA as a confounder and ignore its role as a potential effect modifier. Doing so in the current study would have prevented us from finding that SB is only related to certain metabolic syndrome components in people with higher MVPA. A third major strength of the current study was the decision to fully examine and describe a possible non-linear relationship between SB and the dependent variables, rather than performing transformations or categorising our dependent variables. Although none of the relationships proved to be non-linear, this is not 
something we could have detected without investigating the possibility.

This study also has several limitations. One limitation inherent in the use of a hip-mounted accelerometer, such as the ActiGraph, is that its inability to discern sitting from standing still may lead to measurement error in the estimate of time spent in SB. Kozey-Keadle et al found the ActiGraph accelerometer to significantly underestimate sitting time compared with both direct observation [31] and the activPAL [32], a thigh-worn accelerometer that can detect changes in posture; however, the differences were less than $5 \%$. Future studies should aim to verify our findings using similar devices that can differentiate between sitting and standing with higher accuracy. Another limitation of using a hip-mounted accelerometer to measure MVPA is that it does so poorly for certain activities such as cycling and swimming. In contrast, these devices are especially accurate at measuring ambulatory activity, and walking is the most common form of physical activity in US adults [33]. We also deliberately selected an activity count threshold designed to capture daily physical activities of moderate intensity ( $\geq 3$ metabolic equivalents), thus reducing the likelihood of underestimating the amount of relevant activities. Another unavoidable limitation inherent in the cross-sectional design of the current study is the inability to make causal inferences. It is possible that those with the metabolic syndrome or its components were consequently more sedentary rather than the other way around. Finally, although the current study had a large sample size, limited statistical power to detect the number of multiplicative interactions that were investigated remains a possibility.

\section{Conclusion}

We found that total daily time spent in SB, but not breaks in $\mathrm{SB}$, was linearly related with higher odds of the metabolic syndrome in large sample of US adults aged $>18$ years. This relationship was not modified by sex, age or time spent in MVPA. However, the association between total SB and several components of the metabolic syndrome did differ by the MVPA level. These findings suggest that there is no threshold of SB at which the odds of the metabolic syndrome begin to rise. Future studies should investigate this question prospectively to provide causal evidence for a recommendation to maintain low amounts of total time spent in SB so as to lessen the odds of developing the metabolic syndrome.

Funding This research received no specific grant from any funding agency in the public, commercial or not-for-profit sectors.

Duality of interest The authors declare that there is no duality of interest associated with this manuscript
Contribution statement All authors contributed to the design, interpretation of data, and drafting of the article. All authors provided final approval of the version to be published. KPG is the guarantor of this work.

\section{References}

1. Matthews CE, Chen KY, Freedson PS et al (2008) Amount of time spent in sedentary behaviors in the United States, 2003-2004. Am J Epidemiol 167:875-881

2. Network SBR (2012) Letter to the editor: standardized use of the terms "sedentary" and "sedentary behaviours". Appl Physiol Nutr Metab 37: $540-542$

3. Thorp AA, Owen N, Neuhaus M, Dunstan DW (2011) Sedentary behaviors and subsequent health outcomes in adults a systematic review of longitudinal studies, 1996-2011. Am J Prev Med 41:207-215

4. Grundy SM, Cleeman JI, Daniels SR et al (2005) Diagnosis and management of the metabolic syndrome. An American Heart Association/National Heart, Lung, and Blood Institute Scientific Statement. Executive summary. Cardiol Rev 13:322-327

5. Edwardson CL, Gorely T, Davies MJ et al (2012) Association of sedentary behaviour with metabolic syndrome: a meta-analysis. PLoS One 7, E34916

6. Healy GN, Wijndaele K, Dunstan DW et al (2008) Objectively measured sedentary time, physical activity, and metabolic risk: the Australian Diabetes, Obesity and Lifestyle Study (Ausdiab). Diabetes Care 31:369-371

7. Bankoski A, Harris TB, McClain JJ et al (2011) Sedentary activity associated with metabolic syndrome independent of physical activity. Diabetes Care 34:497-503

8. Saidj M, Jørgensen T, Jacobsen RK, Linneberg A, Aadahl M (2013) Separate and joint associations of occupational and leisure-time sitting with cardio-metabolic risk factors in working adults: a crosssectional study. PLoS One 8, E70213

9. Centers For Disease Control And Prevention (CDC), National Center For Health Statistics (NCHS) (2012) National Health And Nutrition Examination Survey: Sample Design, 2011-2014. U.S. Department Of Health And Human Services, Centers for Disease Control and Prevention, Hyattsville, MD. Available from www.cdc.gov/Nchs/ Data/Series/Sr_02/Sr02_162.Pdf, accessed 16 July 2013

10. Gennuso KP, Gangnon RE, Matthews CE, Thraen-Borowski KM, Colbert LH (2013) Sedentary behavior, physical activity, and markers of health in older adults. Med Sci Sports Exerc 45:1493-1500

11. Matthew CE (2005) Calibration of accelerometer output for adults. Med Sci Sports Exerc 37:S512-S522

12. Troiano RP, Berrigan D, Dodd KW, Masse LC, Tilert T, Mcdowell M (2008) Physical activity in the United States measured by accelerometer. Med Sci Sports Exerc 40:181-188

13. Centers for Disease Control and Prevention (CDC), National Center for Health Statistics (NCHS) (2004) National Health and Nutrition Examination Survey: Physician Examination Procedures Manual. U.S. Department Of Health And Human Services, Centers for Disease Control and Prevention, Hyattsville, MD. Available from www.cdc.gov/Nchs/Data/Nhanes/Nhanes_05_06/Pe.Pdf, accessed 19 November 2014

14. Centers for Disease Control and Prevention (CDC), National Center for Health Statistics (NCHS) (2014) National Health and Nutrition Examination Survey Laboratory Protocol. U.S. Department of Health and Human Services, Centers for Disease Control and Prevention, Hyattsville, MD. Available from www.cdc.gov/nchs/nhanes/ nhanes2005-2006/lab methods 05 06.htm, accessed 19 November 2014 
15. Healy GN, Dunstan DW, Salmon J et al (2008) Breaks in sedentary time: beneficial associations with metabolic risk. Diabetes Care 31:661-666

16. Henson J, Yates T, Biddle SJ et al (2013) Associations of objectively measured sedentary behaviour and physical activity with markers of cardiometabolic health. Diabetologia 56:1012-1020

17. Healy GN, Matthews CE, Dunstan DW, Winkler EA, Owen N (2011) Sedentary time and cardio-metabolic biomarkers in US adults: NHANES 2003-06. Eur Heart J 32:590-597

18. Cooper AR, Sebire S, Montgomery AA et al (2012) Sedentary time, breaks in sedentary time and metabolic variables in people with newly diagnosed type 2 diabetes. Diabetologia 55:589-599

19. Scheers T, Philippaerts R, Lefevre J (2013) Sensewear-determined physical activity and sedentary behavior and metabolic syndrome. Med Sci Sports Exerc 45:481-489

20. Physical Activity Guidelines Advisory Committee (2008) Physical activity guidelines advisory committee report, 2008. U.S. Department of Health and Human Services, Washington, DC

21. Lakka TA, Laaksonen DE, Lakka HM et al (2003) Sedentary lifestyle, poor cardiorespiratory fitness, and the metabolic syndrome. Med Sci Sports Exerc 35:1279-1286

22. Ford ES, Kohl HW, Mokdad AH, Ajani UA (2005) Sedentary behavior, physical activity, and the metabolic syndrome among U.S. adults. Obes Res 13:608-614

23. Bertrais S, Beyeme-Ondoua JP, Czernichow S, Galan P, Hercberg S, Oppert JM (2005) Sedentary behaviors, physical activity, and metabolic syndrome in middle-aged French subjects. Obes Res 13:936-944

24. Hamilton MT, Hamilton DG, Zderic TW (2004) Exercise physiology versus inactivity physiology: an essential concept for understanding lipoprotein lipase regulation. Exerc Sport Sci Rev 32:161-166

25. Hamilton MT, Hamilton DG, Zderic TW (2007) Role of low energy expenditure and sitting in obesity, metabolic syndrome, type 2 diabetes, and cardiovascular disease. Diabetes 56:2655-2667

26. Stump CS, Hamilton MT, Sowers JR (2006) Effect of antihypertensive agents on the development of type 2 diabetes mellitus. Mayo Clin Proc 81:796-806

27. Bey L, Hamilton MT (2003) Suppression of skeletal muscle lipoprotein lipase activity during physical inactivity: a molecular reason to maintain daily low-intensity activity. J Physiol 551:673-682

28. Megeney LA, Neufer PD, Dohm GL et al (1993) Effects of muscle activity and fiber composition on glucose transport and Glut-4. Am J Physiol 264:E583-E593

29. Hamilton MT, Hamilton DG, Zderic TW (2014) Sedentary behavior as a mediator of type 2 diabetes. Med Sport Sci 60:11-26

30. Zderic TW, Hamilton MT (2012) Identification of hemostatic genes expressed in human and rat leg muscles and a novel gene (LPP1/PAP2A) suppressed during prolonged physical inactivity (sitting). Lipids Health Dis 11:137

31. Kozey-Keadle S, Libertine A, Lyden K, Staudenmayer J, Freedson PS (2011) Validation of wearable monitors for assessing sedentary behavior. Med Sci Sports Exerc 43:1561-1567

32. Kozey-Keadle S, Libertine A, Staudenmayer J, Freedson P (2012) The feasibility of reducing and measuring sedentary time among overweight, non-exercising office workers. J Obes 2012:282303

33. Chodzko-Zajko WJ, Proctor DN, Fiatarone Singh MA et al (2009) American College of Sports Medicine position stand. Exercise and physical activity for older adults. Med Sci Sports Exerc 41:1510-1530 